\title{
INSERÇÃO DO PAI NAS MATERNIDADES MUNICIPAIS DO RECIFE: OPINIÃO DOS TÉCNICOS E AUXILIARES DE ENFERMAGEM
}

Renato Daniel Melo da Silva¹, Luiziane Suza Vasconcelos de Lima¹, Delmilena Maria Ferreira Aquino', Leonardo Xavier Lima e Silva ${ }^{1}$ Amanda Bezerra da Silva ${ }^{1}$, Cynthia Dantas Vicente ${ }^{1}$

Objetivo: descrever a opinião dos técnicos e auxiliares de enfermagem do centro obstétrico e alojamento conjunto, sobre a inserção do pai como acompanhante no processo parturitivo, nas maternidades municipais do Recife. Metodologia: pesquisa quantitativa, desenvolvida no mês de agosto de 2016, através de questionário, em três maternidades municipais do Recife, com 80 técnicos e auxiliares de enfermagem. Resultados: foi identificado que $25 \%$ dos respondentes não concordam com a inserção do pai no processo parturitivo, e $75 \%$ dos profissionais consideraram que o pai pode ajudar de alguma forma no processo parturitivo. Observou-se que a falta de orientação aos pais (50\%) dificultaria esta inserção. Conclusão: os resultados desta pesquisa indicam para a necessidade de aprofundar as discussões no contexto estudado.

Descritores: humanização, parto humanizado, acompanhantes, equipe de enfermagem

\section{INSERTION OF THE FATHER IN THE MUNICIPALITY OF RECIFE: OPINION OF THE TECHNICIANS AND NURSING ASSISTANTS}

Objective: to describe the opinion of nursing technicians and assistants of the obstetric center and joint accommodation, on the insertion of the father as companion in the parturition process, in the municipal maternity hospitals of Recife. Methodology: quantitative research, developed in August 2016, through a questionnaire, in three maternity hospitals in Recife. The sample consisted of 80 technicians and nursing assistants. Results: It was identified that $25 \%$ of the respondents did not agree with the father's insertion in the parturitive process, and $75 \%$ of the professionals considered that the father could help in some way in the parturitive process. It was observed that lack of orientation to parents (50\%) would make this insertion difficult. Conclusion: the results of this research indicate the need to deepen the discussions in the studied context.

Descriptors: humanization, humanized birth, companions, nursing staff

\section{INSERCIÓN DE LAMATERNIDAD EN PADRE MUNICIPAL DE CORAL: OPINIÓN DE LANATURALEZA DE LAAUXILIAR TÉCNICO Y DE ENFERMERÍA}

Objetivo: Describir las opiniones de los técnicos y auxiliares de enfermería en la sala de partos y el alojamiento conjunto durante la inserción del padre como compañero en el proceso del parto, las maternidades municipales en Recife. Metodología: La investigación cuantitativa realizada en agosto de 2016, a través de un cuestionario en tres hospitales municipales de Recife, com 80 técnicos y auxiliares de enfermería. Resultados: se identificó que el 25\% de los encuestados no está de acuerdo con la inserción de su padre en el proceso del parto, y el 75\% de los encuestados consideró que el padre puede ayudar de alguna manera en el proceso del parto. Se observó que la falta de orientación para los padres (50\%) dificultan este inserto. Conclusión: Los resultados de esta investigación indican la necesidad de nuevas discusiones en el contexto estudiado.

Descriptores: humanización, parto humanizado, cuidadores, personal de enfermería 


\section{INTRODUÇÃO}

A assistência prestada à mulher no parto e nascimento vem sofrendo diversas modificações ao longo dos tempos, decorrentes da institucionalização desses acontecimentos e dos avanços tecnológicos e científicos na área da obstetrícia. Com isso, esses eventos deixaram de acontecer no âmbito familiar, em que as pessoas estavam ligadas por fortes vínculos familiares e suportes sociais e passaram a ocorrer em instituições hospitalares, com o controle do parto e do comportamento da parturiente..$^{(1)}$

A partir desta visivel necessidade de mudança na atenção ao parto, em 1980, iniciou-se um movimento organizado para proporcionar uma assistência apropriada e de qualidade à parturiente. No Brasil, este movimento recebeu a denominação de humanização do parto, em cujo modelo de assistência são resgatados valores como o protagonismo, a individualidade, a privacidade $\mathrm{e}$ a autonomia de cada mulher. Ele envolve práticas cujo objetivo é promover partos fisiológicos, eliminando-se as intervenções desnecessárias e oferecendo outras comprovadamente consideradas benéficas. O modelo de humanização do parto pressupõe que segurança não é sinônimo de intervenção e tecnologia. Ao contrário, pressupõe a mínima utilização de intromissão no processo fisiológico de nascimento. Dentre as práticas que foram preconizadas, há a possibilidade da parturiente escolher a pessoa que a acompanhará na maternidade, conforme recomendam o Ministério da Saúde e a Organização Mundial da Saúde (OMS).(2)

Um dos resultados desta mobilização, em 2005, foi a aprovação, no Brasil, da Lei n. 11.108/2005, que garante à parturiente o direito de ter a presença de um acompanhante de livre escolha, durante o trabalho de parto, parto e pósparto imediato, nos serviços que compõem o Sistema Único de Saúde - SUS, da rede própria ou conveniada. ${ }^{(3)}$

A presença de um acompanhante escolhido pela parturiente durante $\mathrm{o}$ processo de parto contribui significativamente para a prestação de apoio durante este, proporcionando bem-estar físico e emocional à mulher, oferecendo conforto e encorajamento, o que permite reduzir os sentimentos de solidão, ansiedade, e os niveis de estresse causados pela vulnerabilidade, assim como uma boa evolução dos períodos clínicos do parto. Além disso, o acompanhante passa segurança durante todo o processo parturitivo, o que pode diminuir as complicações na gestação, parto e puerpério, a utilização de analgesia, ocitocina, cesarianas e tempo de hospitalização do binômio mãe-filho. (4)

Por tratar-se de um processo de transição maturacional e social, os seres humanos que o vivenciam podem necessitar de assistência profissional. Os profissionais de saúde, neste contexto, atuam no sentido de facilitar a transição, buscando promover o desenvolvimento humano e a vida em sua plenitude. A consolidação deste acontecimento ocorre por meio do parto, que, por sua vez, é um processo abrupto que causa rapidamente mudanças fisiológicas e psicológicas intensas na mulher. (1)

Considerando este contexto da humanização da assistência ao parto e nascimento, optou-se por desenvolver um estudo cujo objetivo é descrever a opinião dos técnicos de enfermagem do centro obstétrico e alojamento conjunto, sobre a inserção do pai como acompanhante no processo parturitivo, em maternidades municipais do Recife.

\section{MÉTODO}

Estudo quantitativo do tipo descritivo. A pesquisa foi desenvolvida na cidade do Recife, nas maternidades de risco habitual: Prof. Arnaldo Marques, Prof. Bandeira Filho e Prof. Barros Lima que são unidades públicas, municipais, que atendem a demanda SUS, realizado com funcionários do centro obstétrico e alojamento conjunto, que aceitaram em participar voluntariamente do estudo, após assinatura do Termo de Consentimento Livre e Esclarecido.

A amostra foi delimitada com o auxílio da Calculadora Estatística Sample Size Calculator Raosoft. Para efeito de estimativa de população, utilizou-se a média de número de técnicos e auxiliares de enfermagem escalados nos setores da pesquisa, que corresponderam a aproximadamente 180 funcionários atuantes nas maternidades municipais do Recife.

Considerando que foram analisadas múltiplas variáveis com prevalências distintas, adotou-se uma estimativa de prevalência de $50 \%$, intervalo de confiança de $95 \%$ e margem de erro de cinco pontos percentuais, conforme a fórmula proposta na calculadora de estatística. O cálculo amostral correspondeu a 123 sujeitos.

No presente estudo a amostragem foi do tipo conveniência. Como critérios de inclusão, foram estabelecidos os seguintes itens: estar lotado na maternidade há mais de um ano; aceitar livremente participar da pesquisa, mediante assinatura do termo de consentimento livre e esclarecido. Os critérios de exclusão considerados foram: o funcionário que estivesse de férias, licença prêmio ou médica durante o período da coleta de dados.

Inicialmente, a amostra proposta do estudo foi de 123 profissionais técnicos e auxiliares de enfermagem das maternidades, contudo, 28 profissionais se recusaram a participar, 10 estavam a menos de um ano nos setores 
da coleta, 05 estavam de férias ou afastados por licença médica e/ou prêmio, restando apenas 80 profissionais que aceitaram participar deste estudo.

A coleta dos dados foi realizada durante o mês de agosto de 2016, através da aplicação de um questionário ao profissional que aceitou participar da pesquisa. Foi utilizado um questionário elaborado pelos autores, contemplando 16 perguntas objetivas, contendo variáveis relativas aos dados do perfil profissional, participação do pai como acompanhante, impactos com a inserção do pai, fatores pessoais e profissionais que impediam a inserção do pai.

As questões éticas foram tratadas conforme a Resolução 466/12 do Conselho Nacional de Saúde. O projeto de pesquisa foi submetido pelo Comitê de ética em Pesquisa do Instituto de Medicina Integral Professor Fernando Figueira - IMIP/PE, aprovado pelo CAAE: 56538316.0.0000.5201, parecer consubstanciado $\mathrm{n}$ ㅇ. 1.634 .781 .

\section{RESULTADOS}

Nas maternidades municipais, foram analisados 80 profissionais, do total de respondentes apenas 8,7\% eram do sexo masculino. Todos possuíam no mínimo o ensino médio completo, sendo que $6 \%$ já haviam concluido algum curso superior. A grande maioria tem entre 11 e 30 anos (86\%) de atividade profissional, e entre 6 e 20 anos (61\%) de experiência em maternidade. A maioria trabalha no turno diurno (63,7\%). Há uma concentração maior de participantes com idade acima dos 40 anos o que correspondeu a 69\% da amostra.

Quanto à inserção do pai como acompanhante no préparto, parto e pós-parto $(n=80), 25 \%$ dos respondentes não concordam com essa participação em nenhum dos momentos do processo de parto e nascimento, 61,3\% concorda com ela em alguns momentos, e 13,7\% dos profissionais manifestaram apoio e estímulo para inserção do pai no cenário obstétrico em todos os momentos.

Dentre os participantes, $75 \%$ assinalou que o pai pode ajudar de alguma forma no processo de parto e pós-parto, enquanto $25 \%$ percebem esta inserção de forma negativa. É possivel observar certa tendência de escolha dos respondentes pelas formas de apoio paterno relacionado ao aspecto emocional, tendo em vista que o suporte emocional: segurar a mão, realizar massagem e fortalecer vínculo mãe-filho, aparecem como as mais frequentes. Curiosamente, abraçar/beijar, que também pode ser considerado um apoio emocional, aparece com expressão mínima (6,2\%), talvez devido à conotação sexual dessa forma de suporte emocional, sendo ainda tabu na assistência ao trabalho de parto e parto.
Quanto a possíveis repercussões positivas da presença do pai no processo de parto e pós-parto, considerando toda a amostra ( $N=80), 25 \%$ respondeu que não existem repercussões, enquanto $36,2 \%$ assinalou que só há repercussão positiva para a mulher, e $38,7 \%$ respondeu que há tanto para a mulher quanto para o profissional.

Em relação aos fatores impeditivos que levariam técnicos de enfermagem a se opor à inserção do pai como acompanhante, observa-se que há predominância dos fatores pessoais estresse (86\%) e medo (58\%), e do fator falta de orientação aos acompanhantes (50\%), como aspecto profissional que dificultaria esta inserção

Quanto à existência de infraestrutura na maternidade para que o pai seja inserido nos processos de parto, nascimento e pós-parto, $83 \%$ considerou não haver disponibilidade de recursos, 17\% respondeu que há, porém com deficiência de recursos físicos ou materiais, e nenhum dos respondentes assinalou que sim, haveria condições suficientes para recepção e alojamento do acompanhante

\section{DISCUSSÕES}

A predominância feminina na enfermagem é explicada em função de seu arquétipo. Este atributo está presente em várias culturas, entre os cuidadores, ao longo do tempo, no cuidado dos doentes, como extensão do trabalho da mulher. ${ }^{(5)}$

A presença do pai não é muito bem aceita durante o processo de parto e nascimento, por sua vez, ficando assim à mercê da decisão dos profissionais, uma vez que se estabelece uma relação de poder sobre o parto e o corpo da mulher.(4) Este achado reflete que $25 \%$ dos profissionais desta amostra, esperam da parturiente a aceitação das circunstâncias, inerente ao modelo de assistência tecnicista, centrado na conveniência do profissional de saúde e da instituição, e não nas necessidades da mulher

A formação dos técnicos e auxiliares de enfermagem por ser mais voltada para desempenho de funções tecnicistas, parece ter pouca ênfase no que diz respeito à humanização da assistência. Talvez este treinamento inadequado justifique que apenas 13,7\% dos respondentes aceite a inserção do pai em todo o cenário obstétrico. É importante ressaltar que a implementação de projetos com a inserção do pai requer uma preparação prévia dos profissionais de saúde, bem como conexão com uma proposta ampliada de assistência humanizada ao parto e nascimento. Neste sentido, faz-se necessário que tais profissionais transformem sua prática na direção de uma relação mais humanizada e segura, onde o parto possa ser resgatado como o momento do nascimento, respeitando todos os seus significados e devolvendo à mulher o legítimo direito de ser mãe com humanidade e segurança. ${ }^{(6-8)}$ 
O papel do acompanhante é definido como elemento fundamental para dar suporte emocional. É uma das maneiras de a mulher encontrar forças para levar o trabalho de parto e parto de forma mais tranquila, diminuindo a ansiedade, e, assim, tornar o nascimento o mais "natural" possivel.(1) Nesta direção, a presença do acompanhante é vista como necessidade para apoiar a parturiente, aliviar o desconforto decorrente do processo parturitivo e garantir sua segurança, além de ser um marco para a convivência entre a mulher e a pessoa escolhida. ${ }^{(4)}$

Os profissionais deste estudo que atuam na assistência ao parto e nascimento avaliam de forma positiva a presença do acompanhante, reconhecem os benefícios de sua contribuição na fisiologia do parto e melhoria na qualidade da assistência, quando comparados a outros estudos. $O$ apoio por uma única pessoa durante o parto mostrou que o apoio físico, empático e contínuo, durante o trabalho de parto, apresentava benefícios, dentre eles, um trabalho de parto mais curto, um menor número de intervenções desnecessárias. ${ }^{(7,9)}$

Mesmo sem qualquer sensibilização dos técnicos e auxiliares de enfermagem deste estudo para receber esse novo personagem no cotidiano do atendimento ao processo de parto e nascimento, eles podem aceitar a proposta e incorporá-la de forma positiva no desenvolvimento de suas atividades assistenciais, conforme $38,7 \%$ dos participantes. Por outro lado, a deficiência na formação técnica, déficit de atualizações e sensibilizações podem gerar o despreparo/ insegurança do profissional para desenvolver a assistência junto ao pai,(10,11) fatores que podem ter influenciado a resposta de $61,2 \%$ dos respondentes. A inclusão deste novo agente no parto deve ser estimulada e valorizada desde o início da gestação, na medida em que esse investimento contínuo asseguraria um maior preparo do acompanhante tendendo a produzir resultados positivos em diferentes dimensões do processo reprodutivo. ${ }^{(9)}$

É preciso garantir o acesso às melhores práticas assistenciais disponíveis a partir do conhecimento atual e reduzir o grave e generalizado distanciamento entre as práticas de atenção ao parto no Brasil e as recomendações baseadas em evidências científicas. Um exemplo seria justamente inserir o acompanhante durante o trabalho de parto, o que é dificultado sob diversas justificativas, como a falta de estruturas físicas, déficit de recursos humanos e de insumos, mesmo que tal inserção seja um direito garantido há mais de uma década, por meio da Lei 11.108/2005, cujo cumprimento é ainda incipiente no país. ${ }^{(6,12)}$ Ao que parece, as instituições hospitalares ainda não estão em condições de acolher adequadamente a mulher para a parturição e as demandas geradas de acordo com suas escolhas, necessidades e desejos individuais e culturais, como sugerem outros estudos. . $^{(710)}$

Como limitações do estudo, pontuamos o fato de que o questionário foi respondido durante o momento de trabalho dos técnicos e auxiliares de enfermagem, implicando a presença de outros profissionais no mesmo espaço, o que pode ter interferido de algum modo em suas respostas. Além disso, o próprio questionário fechado, como instrumento quantitativo, apresenta limitações quanto a uma compreensão mais aprofundada no que se diz respeito à opinião dos citados profissionais. Em futuras pesquisas, seria interessante a utilização de outros métodos e técnicas de inspiração mais qualitativa, a fim de complementar e enriquecer os dados colhidos. Finalmente, o estudo realizado apresentou limitações importantes no que se refere ao tamanho da amostra, uma vez que, ao ser reduzida em praticamente um terço do quantitativo fornecido pelo cálculo amostral, leva a pensar se a inclusão dos que se recusaram a participar, por exemplo, poderia modificar o padrão dos resultados encontrados. Além disso, convém destacar que a amostra foi composta por profissionais de apenas três das maternidades existentes na cidade de Recife-PE, limitando sua abrangência inferencial.

\section{CONSIDERAÇÕES FINAIS}

A análise da opinião dos técnicos e auxiliares de enfermagem das maternidades municipais estudadas sobre a presença do pai durante o processo parturitivo permitiu compreender que esta figura foi valorizada de forma superficial por parte de alguns profissionais, tendo em vista os reais benefícios que o pai poderá proporcionar para a mulher, com destaques para o suporte emocional, e a autoconfiança das parturientes/puérperas.

Os resultados desta pesquisa indicam a necessidade de aprofundar as discussões no contexto estudado, para a reflexão sobre a percepção relativa à figura do pai como acompanhante no processo do parto e nascimento, com vistas às mudanças na prática clínica, pois a rejeição por parte dos profissionais tende a desaparecer quando estes são incluídos em processos educativos os quais devem ser promovidos para a sensibilização do grupo

Faz-senecessário investimentos naimplementação das boas práticas, baseadas em evidências científicas, em serviços que atendem em todo processo gravídico puerperal, o que resultaria em formação profissional com visões humanísticas, infraestrutura adequada para atender a demanda das maternidades, e avaliação de modelos assistenciais com atuação em equipe multidisciplinar, nos aspectos socioculturais como o protagonismo da mulher $e$ o apoio contínuo à mulher no parto e nascimento. 
Sugere-se a realização de fóruns locais para a discussão coletiva das evidências científicas nacionais e internacionais disponíveis, além das experiências exitosas de implementação da lei do acompanhante no cenário da parturição. Acrescenta-se o fato da importância de inclusão do pai como parte da filosofia e missão institucional, para assim potencializar a mudança de paradigma da atenção ofertada à mulher em processo parturitivo e sua família.

Espera-se que os resultados desta investigação possam contribuir com a melhoria da assistência, através de ações educativas de sensibilização para a equipe de enfermagem com intuito de fortalecer e estimular a presença do pai como acompanhante neste momento tão especial na vida da mulher.

Propõe-se, ainda, a execução de novos estudos acerca do tema, tendo em vista comparar as diversas opiniões dos profissionais entre cada maternidade municipal a fim de obter uma amostra mais ampliada e melhorar a identificação do fenômeno.

\section{REFERÊNCIAS}

1. Dodou HD, Rodrigues DP, Guerreiro EM, Guedes MVC, Lago PN, Mesquita NS. A contribuição do acompanhante para a humanização do parto e nascimento: percepções de puérperas. Esc Anna Nery [Internet]. 2014[citado em 20 out 2016]; 18(2): 262-269. Disponivel em: http://www.scielo.br/scielo.php?script=sci_arttextEpid=S1414-81452014000200262.

2. Organização Mundial da Saúde (OMS). Maternidade segura: assistência ao parto normal. Um guia prático. Brasilia: Organização Mundial da Saúde; 1996.

3. Brasil. Lei № 11.108, de 7 de abril de 2005. Dispõe sobre a garantia às parturientes o direito à presença de acompanhante durante o trabalho de parto, parto e pós parto imediato, no âmbito do Sistema Único de Saúde - SUS. Diário Oficial da República Federativa do Brasil, 8 abril 2005. Seção 1.

4. Souza SRRK, Gualda DMR. A experiência da mulher e de seu acompanhante no parto em uma maternidade pública. Texto Contexto Enferm [Internet]. 2016 [citado em $18 \mathrm{dez} 2017$ ]; 25(1):1-9. Disponivel em: http://www.scielo.br/scielo.php?script=sci_arttextEpid=S010407072016000100309\&lng=pt\&tlng=pt

5. Souza, LL, Araujo DB, Silva DS, Bêrredo VCM. Representações de gênero na prática de enfermagem na perspectiva de estudantes. Ciências \& Cognição [Internet]. 2014 [citado em 28 mar 2017]; 19(2): 218-232. Disponivel em: http://cienciasecognicao. org/revista/index.php/cec/article/download/908/pdf_13.

6. Pereira SS, Santos JBS, Carvalho MCMP. Parto natural: a atuação do enfermeiro diante da assistência humanizada. Actas de Saude Colet. [Internet]. 2016 [citado em 18 dez 2017]; 10(3):199213. Disponivel em:http://www.tempusactas.unb.br/index.php/ tempus/article/view/1727.
7. Feijão LBV, Boeckmann LMM, Melo MC. Conhecimento de enfermeiras residentes acerca das boas práticas na atenção ao parto. Enferm Foco [Internet]. 2017 [citado em 18 dez 2017]; 8(3): 35-39. Disponivel em : http://revista.portalcofen.gov.br/index. php/enfermagem/article/view/1318/396

8. Ministério da Saúde (BR). Secretaria de Políticas de Saúde. Área Técnica de Saúde da Mulher. Comissão Nacional de Incorporação de Tecnologias em Saúde. Diretrizes Nacionais de Assistência ao Parto Normal. Brasília: Ministério da Saúde, 2016.

9. Campanati, FLS. Participação paterna no ciclo gravidico puerperal: vivências e sentimentos [monografia]. Ceilândia: Universidade de Brasilia, Faculdade de Ceilândia; 2015 [citado 27 mar 2017]. Disponivel em: http://bdm.unb.br/bitstream/10483/10936/1/2015_FernandaLeticiadaSilvaCampanati. pdf.

10. Brüggemann OM, Ebsen ES, Oliveira ME, Gorayeb MK, Ebele RR. Motivos que levam OS serviços de saúde a não permitirem acompanhante de parto: discursos de enfermeiros. Texto Contexto Enferm [Internet].2014 [citado 29 mar 2017]; 23(2): 270-7. Disponivel em: http://www.redalyc.org/articulo. oa?id=71431352006.

11. Santos RAA, MCP Melo, Cruz DD. Trajetória da humanização do parto a partir de uma revisão integrativa de literatura. Cad. Cult. Ciênc. [Internet] 2015 [citado 26 mar 2017]13(2): 76-89. Disponivel em: http://periodicos.urca.br/ojs/index.php/cadernos/ article/view/838/pdf_1.

12. Lansky S, Freiche AAL, Silva AAM, Campos, D, Bittencourt DAS, Carvalho ML, et al. Mortalidade Neonatal e Avaliação da Assistência Materno-Infantil. Cad. Saúde Pública, Rio de Janeiro, 2014 [citado em 20 jan 2017]. Disponivel em: http://www.scielo. $\mathrm{br} / \mathrm{pdf} / \mathrm{csp} / \mathrm{v} 30 \mathrm{sl} / 0102-311 \mathrm{X}-\mathrm{csp}-30$-sl-0192.pdf 